\title{
TAG Lens: Revolutionizing Optical Microscopy With Ultra-High Speed Variable Focus
}

\author{
C. Theriault ${ }^{1}$, J. Guttenfelder ${ }^{1}$ C.B. Arnold ${ }^{1,2}$ \\ ${ }^{1 .}$ TAG Optics Inc., PO Box 1572, Princeton, NJ 08542, USA \\ 2. Princeton University, Department of Mechanical and Aerospace Engineering, Princeton, NJ, 08544, \\ USA
}

The Tunable Acoustic Gradient Lens, or TAG Lens, is a revolutionary innovation that uses sound waves in liquid to provide the fastest variable focus lens in the field of adaptive optics. Capable of scanning a wide range of focal lengths in microseconds, the TAG lens is orders of magnitude faster than other liquid lenses, optical membrane or mechanical scanning approaches presently available on the market [1]. The award-winning TAG Lens functions by using a piezoelectric element at the boundary of a cylindrical cavity to excite high frequency acoustic pressure waves in a confined liquid. The corresponding oscillations in liquid density create an index of refraction profile like that of a simple aspherical lens (Figure 1). However, unlike static devices that rely on the deformation of a surface or interface, the TAG Lens is a tunable gradient index of refraction (GRIN) lens that does not require any mechanical motion or redistribution of liquid to change its optical properties, allowing it to modulate focal length at very high speeds $(70 \mathrm{kHz}$ to $1 \mathrm{MHz})$. Furthermore, the aspheric nature of the index of refraction profile results in a tunable lens with little to no spherical aberration, making it an ideal candidate for high-speed, high-precision applications [2].

By using the TAG Lens in applications requiring variable focal lengths, we can overcome many of the limitations of traditional z-scanning technologies and bring new advantages to a range of applications. In the field of optical microscopy, high-resolution imaging requires large aperture lenses in order to collect light from wider angles. The characteristics of the TAG Lens 2.5 model achieves nearly 4 diopters of refractive power at an $11 \mathrm{~mm}$ effective aperture, opening the door to emerging imaging applications such as high throughput scanning, imaging cytometry, and diagnostic point-of-care medical imaging applications (Figure 2 and 3). The TAG Lens HP model is specified to handle power throughputs of up to $100 \mathrm{Watts} / \mathrm{cm}^{2}$ in addition to significant increase of transmission efficiency within the infrared wavelength range. This IR transmission efficiency is necessary to enable new functionalities and innovation in applications such as multi-photon microscopy, semiconductor optical inspection, laser manufacturing and optical coherence tomography. When the TAG Lens is turned off, there is no optical power (i.e. it acts as a window) and it can be added into any optical assembly without changing any focusing parameters - a property unique to the TAG Technology Platform.

The optical properties of the TAG Lens are determined by a number of parameters including radius and symmetry of the cylindrical resonant cavity, length of the lens, density and viscosity of the refractive liquid, static filling pressure, frequency and amplitude of the acoustic driving signal, and type of piezoelectric material. Because of the many design degrees of freedom, the specific optical characteristics of the TAG Lens can be customized to accommodate a diverse range of applications.

\section{References:}

[1] M Duocastella, B Sun, and C B Arnold, "Simultaneous imaging of multiple focal planes for three dimensional microscopy using ultra-high-speed adaptive optics", J. Bio. Opt. 17 (2012), p. 1 
[2] A Mermillod-Blondin, E McLeod, and C B Arnold, "High-speed varifocal imaging with a tunable acoustic gradient index of refraction lens", Opt. Lett. 3318 (2008), p.2148

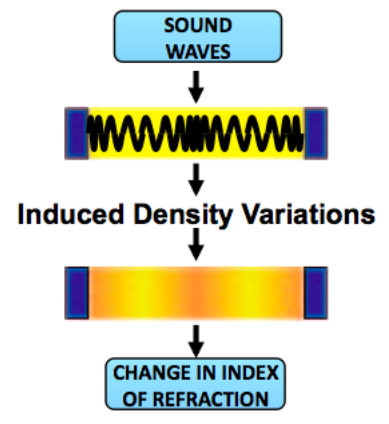

A

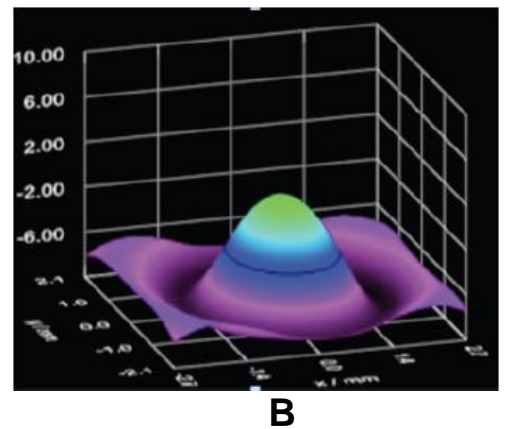

Figure 1. (A) Schematic of the Tunable Acoustic Gradient Lens principle. Sound waves cause the variation in liquid density and corresponding index of refraction that creates the lens effect. (B) Example aspheric wavefront profile

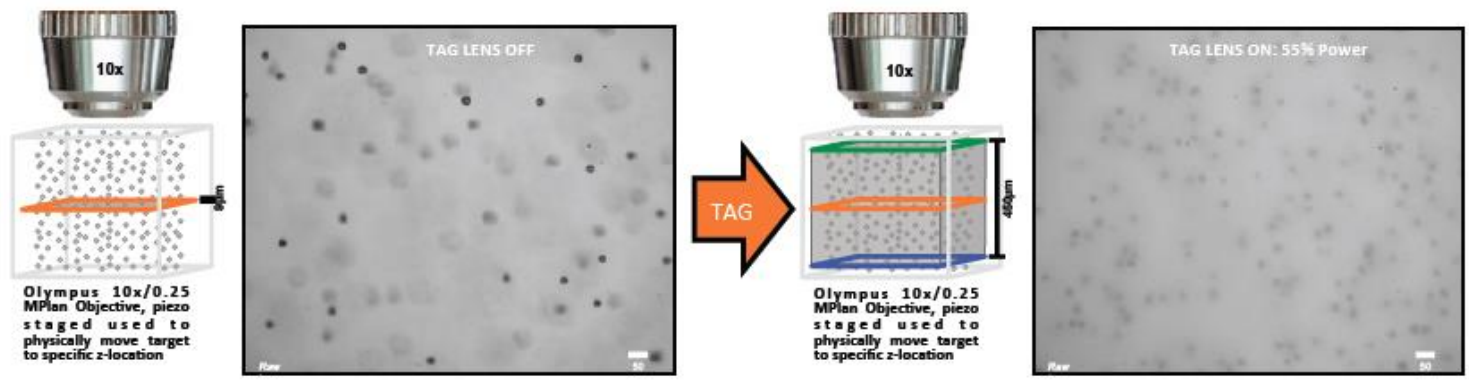

Figure 2. Extended Depth of field application: Particle count in an extended depth of field

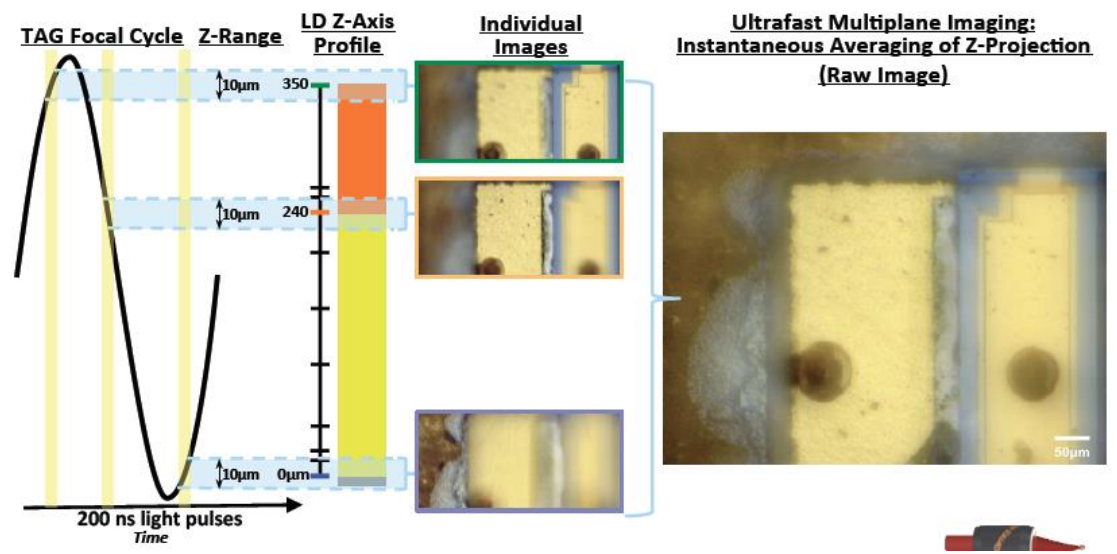

Figure 3: Multi-plane Imaging techniques: 2P microscopy, inspection, etc. 